\title{
Journal of Neurocritical

\section{Rapid Spontaneous Resolution of Contralateral Acute Subdural Hemorrhage Caused by Overdrainage of Chronic Subdural Hemorrhage}

\author{
Minwook Yoo, MD, Jung-Soo Kim, MD, PhD \\ Department of Neurosurgery, Inje University Haeundae Paik Hospital, Busan, Korea
}

Background: Since the first report of a rapidly resolved subdural hemorrhage (SDH) in 1986, few additional case reports have been presented in the literature.

Case Report: An 82-year-old female patient presented with a SDH over the left convexity. The SDH was removed via catheter drainage through a burr hole trephination. Post-operative computed tomography (CT) following $300 \mathrm{~mL}$ drainage from the chronic SDH demonstrated a newly developed SDH along the right convexity. A follow-up CT performed 2 hours later revealed an unexpected significant resolution of the acute SDH.

Conclusion: The spontaneous resolution of acute SDH is believed to result from redistribution by washout of the hematoma by cerebrospinal fluid dilution. However, its exact pathophysiology is not well understood. When surgical evacuation is considered in acute $\mathrm{SDH}$, conservative management should also be considered because spontaneous resolution of hemorrhage remains a possibility.

J Neurocrit Care 2018;11(2):119-123

Key words: Hematoma; Hematoma, Subdural; Hematoma, Subdural, Chronic

\author{
Received May 6, 2018 \\ Revised June 22, 2018 \\ Accepted June 24, 2018 \\ Corresponding Author: \\ Jung-Soo Kim, MD, PhD \\ Department of Neurosurgery, Inje \\ University Haeundae Paik Hospital, 875 \\ Haeundae-ro, Haeundae-gu, Busan \\ 48108, Korea \\ Tel: +82-51-797-0840 \\ Fax: +82-51-797-0841 \\ E-mail: jheaj@paik.ac.kr
}

Copyright $\odot 2018$ The Korean Neurocritical Care Society

\section{INTRODUCTION}

Chronic subdural hemorrhage (SDH) is frequently encountered in elderly individuals. " When we perform an operation for a chronic SDH, the hematoma is usually removed with burr hole trephination as well as catheter drainage. Catheter drainage can be associated with complications, such as acute SDH, pneumocephalus, and infection. Here, we describe a patient with cerebrospinal fluid (CSF) redistribution and hemorrhage washout caused by overdrainage of a chronic SDH. We propose that CSF loss can lead to remote hemorrhaging.

\section{CASE REPORT}

An 82-year-old female patient displayed a decrease in consciousness following a fall. The patient's strength was at $4 / 5$ in the right upper and lower extremities. The patient denied any recent or remote head trauma history. Her past medical history included hypertension. The patient did not take any antiplatelet agents or anticoagulants. Laboratory

(cc) This is an Open Access article distributed under the terms of the Creative Commons Attribution Non-Commercial License (http://creativecommons.org/licenses/by$\mathrm{nc} / 4.0$ ) which permits unrestricted non-commercial use, distribution, and reproduction in any medium, provided the original work is properly cited. 
results did not show coagulopathy. A non-contrast computed tomography (CT) performed during the initial evaluation demonstrated an SDH over the left convexity (Fig. 1A). Magnetic resonance imaging and magnetic resonance angiography revealed no abnormalities, other than a chronic SDH with a thickness of $28.6 \mathrm{~mm}$ in the left convexity (Fig. 1B, C)

Burr hole trephination was performed under general anesthesia. The SDH was removed through catheter drainage without saline irrigation. Chronic SDH is usually drained to $150 \mathrm{~mL}$ per day after surgery. However, postoperative CT following $300 \mathrm{~mL}$ of sudden drainage from the chronic SDH presented a newly developed SDH along the right convexity with a $7 \mathrm{~mm}$ midline shift (Fig. 1D). The right SDH showed high and mixed density, which may be seen as an expansion of a small amount of hemorrhage by decompression due to overdrainage of the left SDH. However, neurological improvement was observed after the operation. The patients mental state improved, and the right hemiparesis was found to be better than its pre-
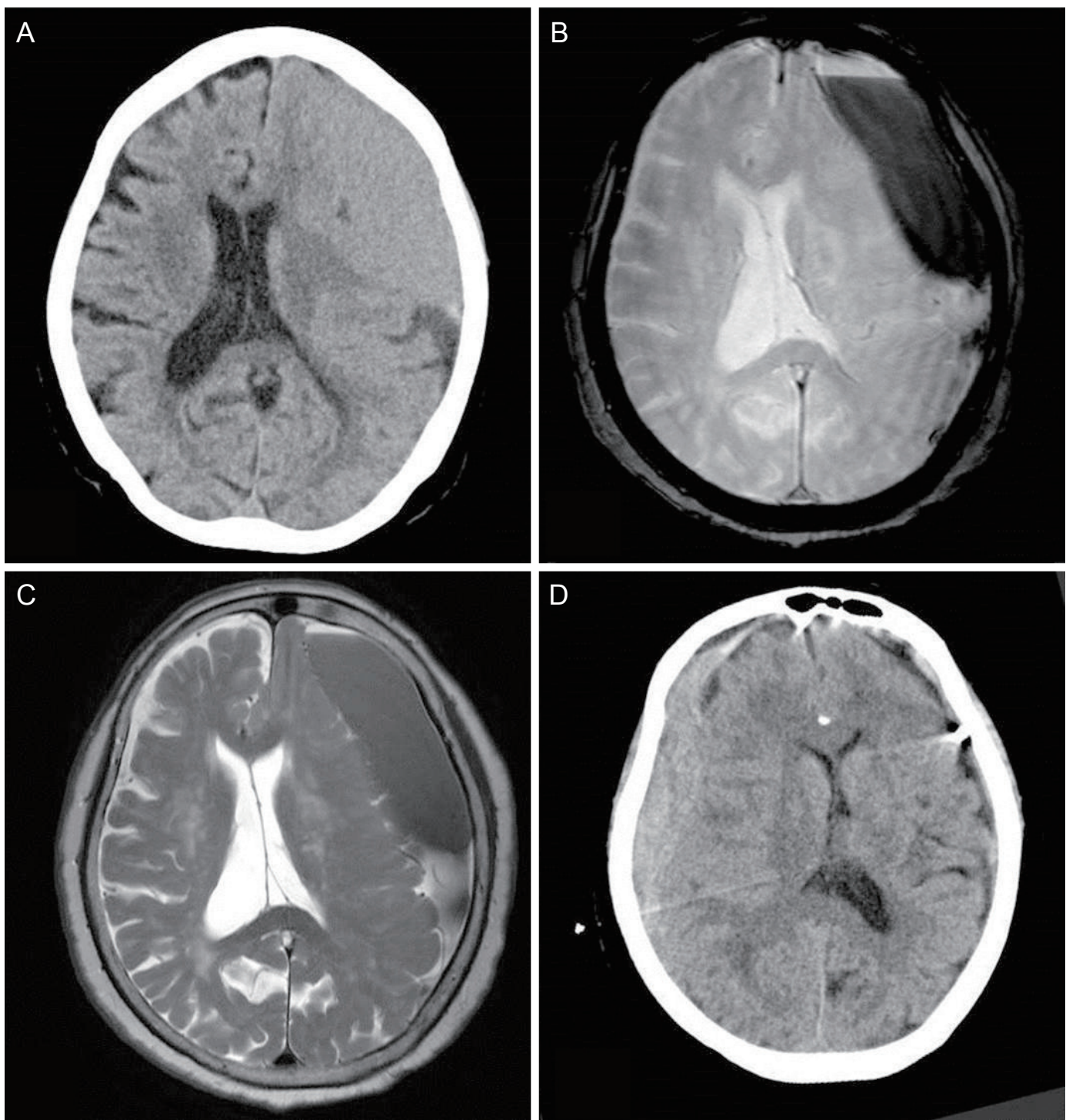

Figure 1. (A) Non-contrast computed tomography demonstrated a SDH over the left convexity. (B) Gradient-echo and (C) T2-weighted images revealing a SDH in the left convexity. (D) Post-operative computed tomography following drainage of the left hematoma, demonstrating a newly developed subdural hemorrhage along the right convexity. SDH, subdural hemorrhage. 
operative state. There was no left side weakness. The catheter was clamped because due to clear drainage in a CSF pattern. The patient was conservatively managed and closely observed. A follow-up CT performed 2 hours later, revealed an unexpected significant resolution of the acute SDH with no midline shifting (Fig. 2A). A CT performed post-operatively on the 2 nd day revealed a more diminished right SDH as well as a lower density in the left SDH (Fig. 2B). A subsequent serial follow-up CT also revealed a gradually decreasing acute SDH in the right convexity (Fig. 2C, D). The patient completely recovered consciousness without experiencing any neurological deficits.
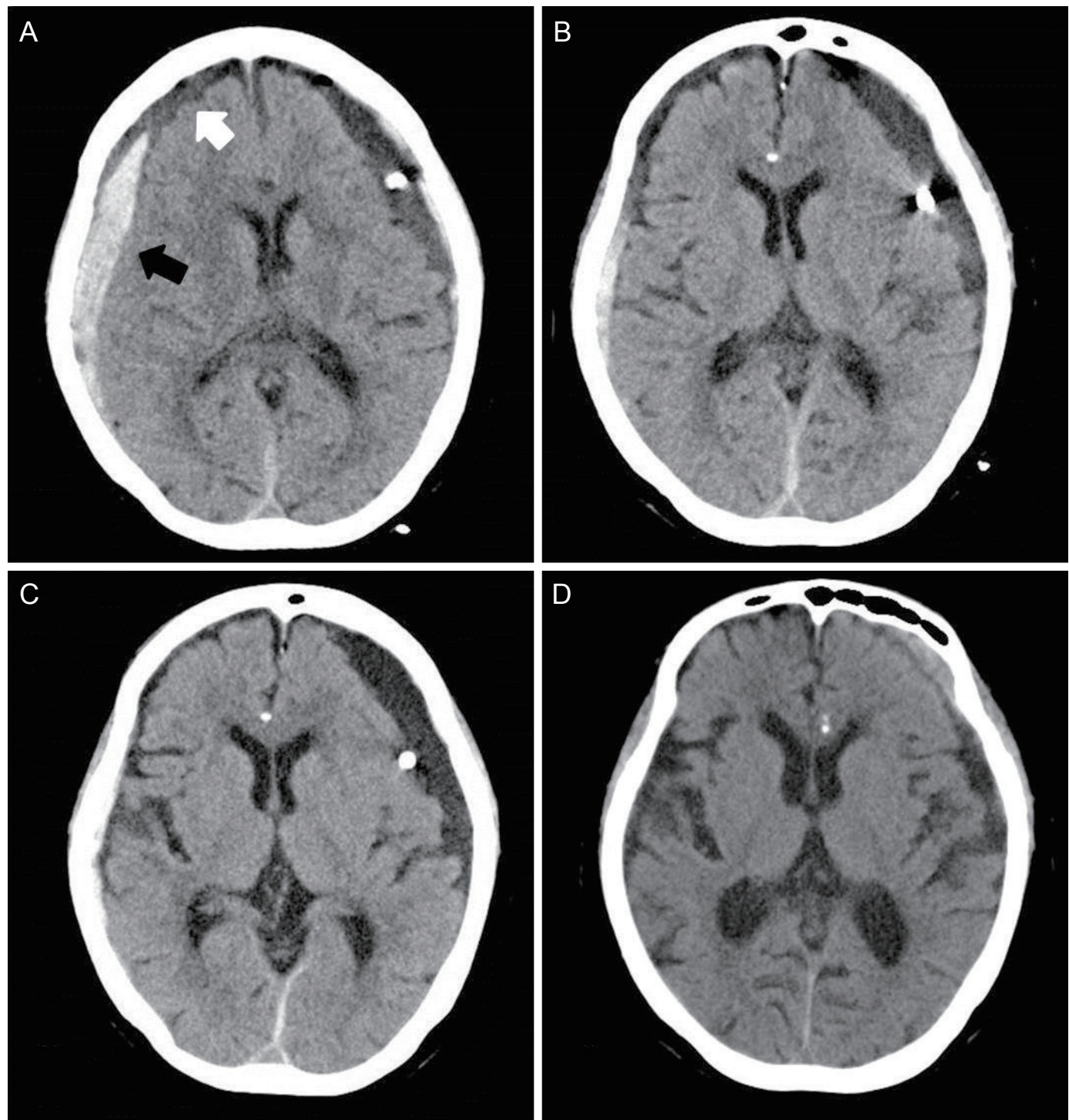

Figure 2. (A) A follow-up CT performed 2 hours later revealed spontaneous resolution of the hemorrhage, and a hyperdense band consistent with bleeding (black arrow), in addition to a layered hypodense component consistent with cerebrospinal fluid (white arrow). (B) A CT performed post-operatively on day 2 revealed a further diminished hemorrhage. (C) A CT performed post-operatively on day 4 revealed a further decreased hemorrhage at the right convexity. (D) A CT performed post-operatively on day 7 revealed little hemorrhages at the right convexity. CT, computed tomography. 


\section{DISCUSSION}

Remote hemorrhage after drainage of a chronic SDH is rarely reported, and the exact pathophysiology is not understood. Several hypotheses have been proposed in order to explain the contralateral SDH. Cohen-Gadol suggested that intracranial hypotension was caused by overdrainage, and remote hemorrhage may occur due to tension in the bridging veins or collapse of the contralateral hemisphere. 'A unique aspect of this report is that the contralateral, hyperacute SDH, caused by overdrainage of the chronic SDH, was resolved.

There have been documented cases of spontaneous resolution of SDH. In all, 40 patients with rapid spontaneous resolution of a SDH have been reported in the literature. ${ }^{2}$ There was no known the mechanism, but several possibilities have been suggested. One theory involving dilution was proposed by Niikawa et al. ${ }^{3}$ It is thought that the SDH might involve a tear in the arachnoid membrane, facilitating the retrograde flow of CSF into the hematoma, resulting in a blood clot washout. Cerebral atrophy has also been shown to facilitate SDH resolution in a theory proposed by Kato et al. ${ }^{4}$ Cerebral atrophy contributes to preventing the formation of well-organized blood clots, making the region more susceptible to washout, via CSF dilution. Redistribution of the hemorrhage towards another subdural space may be another acceptable explanation, as proposed by Bortolotti et al. ${ }^{5}$ The redistribution of the hemorrhage to the spinal subdural space could be one of the mechanisms by which spontaneous resolution can occur, especially in an infratentorial SDH. Although it is not common, if patients complain of a tingling sensation or sensory change as the secondary hemorrhage is decreased, a neurological examination of the spine is recommended.

In this case, the contralateral hyperacute SDH might be resolved via CSF dilution even though its origin was nontraumatic. CT was performed during a neurologically stable state, showing an acute SDH that consisted of two components: a hyperdense band, consistent with bleeding and a layered hypodense component, consistent with
CSF. This data suggests that CSF dilution can prevent the formation of well-organized blood clots. Spontaneous resolution of a SDH is thought to result from redistribution, by washout of the hematoma, via CSF dilution. ${ }^{6}$ Important factors that may be involved in the spontaneous resolution of a SDH appear to include the tearing of the arachnoid membrane and the presence of a wide subdural space into which the hematoma can be redistributed. One limitation of this report is that we could not demonstrate that a SDH redistributed to the spinal subdural space because we did not perform a spinal $\mathrm{CT}$ at the time.

In conclusion, when catheter drainage is being considered for a chronic SDH, the color and amount of post-operative drainage should be closely observed because overdrainage could cause a remote hemorrhage. If the neurological state of the patient is stable with secondary hemorrhage, conservative treatment should be considered due to the possibility of CSF redistribution and hemorrhage washout.

\section{Conflicts of Interest}

The authors of this study have no conflict of interests to disclose.

\section{REFERENCES}

1. Kim CH, Song GS, Kim YH, Kim YS, Sung SK, Son DW, et al. Remote hemorrhage after burr hole drainage of chronic subdural hematoma. Korean / Neurotrauma 2017;13:144-8.

2. Berker M, Gulsen S, Ozcan OE. Ultra-rapid spontaneous resolution of acute posttraumatic subdural hematomas in patient with temporal linear fracture. Acta Neurochir (Wien) 2003; 145:715-7; discussion 717.

3. Niikawa S, Sugimoto S, Hattori T, Ohkuma A, Kimura T, Shinoda J, et al. Rapid reduction of acute subdural hematoma-report of four cases. Neurol Med Chir (Tokyo) 1989;29:8204.

4. Kato N, Tsunoda T, Matsumura A, Yanaka K, Nose T. Rapid spontaneous resolution of acute subdural hematoma occurs by redistribution--two case reports. Neurol Med Chir (Tokyo) 2001:41:140-3. 
5. Bortolotti C, Wang H, Fraser K, Lanzino G. Subacute spinal subdural hematoma after spontaneous resolution of cranial subdural hematoma: causal relationship or coincidence? Case report. / Neurosurg 2004;100(4 Suppl Spine):372-4.
6. Wen L, Liu WG, Ma L, Zhan RY, Li G, Yang XF. Spontaneous rapid resolution of acute subdural hematoma after head trauma: is it truly rare? Case report and relevant review of the literature. Ir J Med Sci 2009;178:367-71. 